\title{
Multiple courses of antenatal corticosteroids for preterm birth study: 2-year outcomes (1)
}

\author{
Asztalos EV, Murphy KE, Hannah ME, Willan AR, Matthews SG, Ohlsson A, Kelly EN, \\ Saigal S, Ross S, Delisle MF, Amankwah K, Guselle P, Gafni A, Lee SK, Armson BA, \\ Sananes $R$, Tomat $L$. \\ Análisis crítico: Jorge Carvajal $\mathrm{C}^{1}$. PhD, Claudio Vera $\mathrm{PG}^{1,2}$. MSc. \\ 1 Unidad de Medicina Materno Fetal, División de Obstetricia y Ginecología, ${ }^{1,2}$ Unidad de Medicina Basada en Eviden- \\ cia; Facultad de Medicina, Pontificia Universidad Católica de Chile.
}

Conflicto de interés: El autor Dr. Claudio Vera, participó como colaborador del estudio MACS en Chile, siendo investigador responsable en la Pontificia Universidad Católica de Chile.

\section{RESUMEN (1)}

Objetivo: El objetivo de este estudio fue determinar el efecto de cursos repetidos de terapia prenatal con corticoesteroides versus placebo en el riesgo de muerte o deterioro neurológico entre los niños enrolados en el estudio Múltiples Cursos de Corticoesteroides Antenatales por Parto Prematuro (MACS), a los 18 a 24 meses de edad. Métodos: Un total de 2305 niños fueron elegibles para el seguimiento; 2104 niños (1069 en el grupo de corticoesteroides prenatales y 1035 en el grupo placebo) fueron monitoreados. El resultado primario fue muerte o deterioro neurológico, definido como parálisis cerebral o retraso cognitivo, a 18 a 24 meses de edad. Los resultados secundarios fueron mediciones de crecimiento (altura, peso y circunferencia craneana). Resultados: Niños expuestos a múltiples cursos de terapia corticoesteroidal prenatal tiene tasas similares de muerte o deterioro neurológico, comparado con niños expuestos a placebo (148 niños [13,8\%] vs. 142 niños [13,7\%]; OR 1,001 [IC 95\% 0,75 a $1,30] ; p=0,95)$. Ellos tuvieron un promedio de peso de $11,94 \mathrm{~kg}$, comparado con $12,14 \mathrm{~kg}$ en el grupo placebo $(p=0,04)$, estatura promedio de $85,51 \mathrm{~cm}$, comparado con $85,46 \mathrm{~cm}(p=0,87)$, y circunferencia craneana promedio de $48,18 \mathrm{~cm}$, comparado con
$48,25 \mathrm{~cm}(p=0,45)$. Conclusiones: La terapia con múltiples cursos de corticoesteroides prenatales, dados cada 14 días, no aumenta ni disminuye el riesgo de muerte o deterioro neurológico a los 18 a 24 meses de edad, comparado con un curso único de terapia corticoesteroidal prenatal. Es necesario continuar el seguimiento de esos niños para medir función neuroconductual, desempeño escolar y posible susceptibilidad a enfermedades.

\section{ANÁLISIS DE LA INVESTIGACIÓN}

\section{A. Relevancia clínica de la investigación}

En embarazadas en riesgo de parto prematuro que han recibido un curso completo de corticoides antenatales para inducción de madurez pulmonar, la administración repetida de cursos de corticoides ¿aumenta en el niño el riesgo de muerte o deterioro neurológico?

Escenario clínico: Los nacidos prematuros se exponen a un elevado riesgo de morbilidad y mortalidad perinatal junto con un elevado riesgo de muerte o retraso neurológico infantil. Se ha demostrado que la administración de corticoides a la mujer embarazada (betametasona $12 \mathrm{mg}$, im., cada 24 horas por dos veces, al menos 48 horas antes del parto), reduce la 
morbilidad y mortalidad perinatal, sin efectos adversos conocidos para la madre y/o el feto (2). Se ha demostrado que este efecto benéfico de los corticoides se mantiene por 7 días desde su administración (3). Por este motivo se sugirió como una buena práctica clínica, la repetición semanal de cursos de corticoides a las mujeres que luego de un primer curso de corticoides, permanecen en riesgo de parto prematuro. Los estudios prospectivos randomizados hasta ahora publicados, permiten sugerir un discreto beneficio de las múltiples dosis de corticoides antenatales (reducción de: síndrome de distrés respiratorio, ductus persistente, uso de surfactante y ventilación mecánica), detectando además una reducción significativa en el peso de nacimiento y circunferencia craneana (4). Estos mismos estudios alertan, sin embargo, sobre las posibles consecuencias a largo plazo del uso de múltiples dosis de corticoides, principalmente respecto de alteraciones del desarrollo neurológico.

\section{B. EL ESTUDIO (1)}

Diseño: Estudio clínico controlado randomizado, en 80 centros repartidos en 20 países. Pacientes: Enroladas en estudio MACS (5) consistentes en 1858 embarazadas (con 2318 fetos) entre 25 y 32 semanas, sin parto luego de 14 a 21 días después de la dosis inicial de corticoesteroides y que se mantenían con riesgo de parto prematuro. Las embarazadas con contraindicación al uso de corticoesteroides, con uso crónico de corticoesteroides, con fetos portadores de anomalías congénitas letales, cursos de corticoesteroides antes de las 23 semanas, o que hubiesen participado en embarazo anterior en el estudio no fueron elegibles como participantes. Se consideró que todos los fetos cuyo resultado perinatal inmediato fue conocido (2304 recién nacidos) eran elegibles para seguimiento a 2 años. Entre ellos, 2104 infantes (1069 en el grupo de corticoesteroides prenatales y 1035 en el grupo placebo) fueron monitoreados. Intervención: 937 embarazadas (1171 fetos) con corticoides antenatales, con dosis repetidas de cursos de betametasona 12 mg i.m. de 2 dosis separadas en 24 horas, cada 2 semanas, hasta las 33 semanas o el parto. Comparación: 921 embarazadas (1147 fetos) recibieron placebo i.m. 2 dosis separadas en 24 horas, cada 2 semanas hasta las 33 semanas o el parto. Resultado primario evaluado: Muerte o deterioro neurológico (parálisis cerebral o retraso cognitivo) a los 18-24 meses de vida. Resultados secundarios evaluados: Peso, talla y circunferencia craneana. Resultados: No se observaron diferencias en ninguno de los componentes individuales del resultado pri- mario compuesto (muerte o deterioro neurológico), la evaluación del desarrollo neurológico no mostró diferencias en niños para la incidencia de parálisis cerebral o retraso cognitivo definido por desviación mayor a 2 desviaciones estándar de la escala de Bayley II. Por otro lado, no se observaron diferencias en los otros resultados evaluados (peso, talla y circunferencia craneana), para lo cual se consideraron diferencias estadísticamente significativas con $p<0,01$. No se observaron diferencias en los subgrupos de neonatos menores de 32 semanas así tampoco en el subgrupo de nacidos antes de 7 días de la última dosis de corticoides (Tabla I).

\section{ANALISIS CRÍTICO}

Validez interna: Estudio con bajo riesgo de sesgo que describe medidas de seguridad implementadas en diversas fases del estudio: criterios de inclusión, randomización, ocultamiento de la secuencia, ciego, seguimiento y análisis. En este caso en particular se trata de un seguimiento planeado de 2 años, con pérdidas de seguimiento menores al $10 \%$ en cada grupo. Si bien los componentes no son de la misma importancia clínica (muerte y deterioro neurológico), tiene sentido analizarlos en conjunto pues solo es evaluable el daño neurológico en los sobrevivientes. Los resultados muestran en forma consistente ausencia de diferencia significativa para la intervención, tanto en el resultado compuesto, como en cada uno de los componentes por separado. El análisis de subgrupos en únicos vs. múltiples, menores de 32 semanas o nacidos con latencia de menos de 7 días, son consistentes con los resultados globales, por lo cual no se visualizan subgrupos en los cuales exista beneficio potencial del uso de dosis repetidas. Destaca que la magnitud de compromiso de talla, circunferencia craneana y peso al nacimiento relacionados al uso de dosis repetidas (hallazgos de los resultados inmediatos del estudio MACs) no se mantiene luego de dos años de vida. Comentario: Estudio de buen diseño y sin fuentes evidentes de sesgo, con apropiado poder estadístico (el mayor conocido entre estudios similares), que no muestra efecto de las dosis repetidas de corticoesteroides antenatales cada 2 semanas, sobre el riesgo de muerte o deterioro neurológico a los dos años de vida, comparado con dosis única. Los efectos encontrados sobre la talla, circunferencia craneana y peso fetal detectados en el período neonatal inmediato han desaparecido. Una revisión sistemática reciente pero con limitaciones metodológicas, sugiere un discreto beneficio de las dosis repetidas de corticoides antenatales, principalmente reduciendo morbilidad respiratoria, aunque 
Tabla I

RESULTADOS PRIMARIO Y SECUNDARIOS DE LA INVESTIGACIÓN

\begin{tabular}{|c|c|c|c|c|}
\hline Resultado & $\begin{array}{l}\text { Dosis repetidas* } \\
\quad(n=1069)\end{array}$ & $\begin{array}{l}\text { Dosis única* } \\
\qquad(n=1035)\end{array}$ & $\begin{array}{c}\text { RRR } \\
(\text { IC 95\%) }\end{array}$ & $\begin{array}{c}\text { RAR } \\
(\text { IC 95\%) }\end{array}$ \\
\hline Compuesto principal & $148(13,8)$ & $142(13,7)$ & $\begin{array}{c}-1 \% \\
(-22 \text { a } 21 \%)\end{array}$ & $\begin{array}{c}-0,001 \\
(-0,03 \text { a } 0,03)\end{array}$ \\
\hline Único & $93 / 675(13,8)$ & $92 / 658(14,0)$ & & \\
\hline Múltiples & $55 / 394(14,0)$ & $50 / 377(13,3)$ & & \\
\hline $\begin{array}{l}\text { Biometría con seguimiento } \\
\text { de } 18 \text { a } 24 \text { meses }\end{array}$ & $\begin{array}{l}\text { Dosis } \\
\text { repetidas* } \\
(n=1020)\end{array}$ & $\begin{array}{c}\text { Dosis } \\
\text { única* } \\
(n=988)\end{array}$ & $\begin{array}{l}\text { Diferencia } \\
\text { promedio } \\
\text { (IC } 95 \%)\end{array}$ & valor $\mathrm{p}$ \\
\hline Peso (g) & 11,94 & 12,14 & $\begin{array}{c}-0,19 \\
(-0,38 \text { a } 0,006)\end{array}$ & 0,04 \\
\hline Talla (cm) & 85,46 & 85,51 & $\begin{array}{c}-0,049 \\
(-0,62 \text { a } 0,53)\end{array}$ & 0,87 \\
\hline $\begin{array}{l}\text { Circunferencia } \\
\text { craneana }(\mathrm{cm})\end{array}$ & 48,18 & 48,25 & $\begin{array}{c}-0,07 \\
(-0,26 \text { a } 0,12)\end{array}$ & 0,45 \\
\hline
\end{tabular}

* Resultados expresados como $\mathrm{n}(\%)$ o promedio. RRR = Reducción relativa de riesgo, RAR= Reducción absoluta de riesgo. Medidas de asociación estimadas a partir de los datos del estudio con el programa CATMAKER® version 1.1 disponible en http://www.cebm.net.

sin lograr una reducción significativa del riesgo de muerte neonatal (4). Este dato, sumado a la ausencia de deterioro neurológico en el seguimiento a dos años (mostrado en este estudio) podría alentar la indicación de dosis repetidas de corticoides. Sin embargo, debemos esperar el resultado de seguimiento a 5 años planeado para estos niños, para conocer si realmente las dosis repetidas de corticoides y las alteraciones antropométricas detectadas al nacimiento, son realmente inocuas. Así también, descartar impacto sobre el desarrollo neurológico. Conclusión: No se recomienda el uso de dosis repetidas de corticoides antenatal para potenciales beneficios a corto plazo, sin tener los resultados de la evaluación de potenciales riesgos a largo plazo y revisiones sistemáticas de mejor calidad.

\section{REFERENCIAS}

1. Asztalos EV, Murphy KE, Hannah ME, Willan AR, Matthews SG, Ohlsson A, Kelly EN, Saigal S, Ross S, Delisle MF, Amankwah K, Guselle P, Gafni A, Lee SK,
Armson BA, Sananes R, Tomat L; Multiple Courses of Antenatal Corticosteroids for Preterm Birth Study Collaborative Group. Multiple courses of antenatal corticosteroids for preterm birth study: 2-year outcomes. Pediatrics 2010;126(5):e1045-55.

2. Roberts D, Dalziel S. Antenatal corticosteroids for accelerating fetal lung maturation for women at risk of preterm birth. Cochrane Database Syst Rev 2006;3:CD004454

3. NIH. Effect of corticosteroids for fetal maturation on perinatal outcomes. NIH Consens Statement 1994;12:1-24.

4. Bevilacqua E, Brunelli R, Anceschi MM. Review and meta-analysis: Benefits and risks of multiple courses of antenatal corticosteroids. J Matern Fetal Neonatal Med 2010;23(4):244-60.

5. Murphy KE, Hannah ME, Willan AR, Hewson SA, Ohlsson A, Kelly EN, Matthews SG,Saigal S, Asztalos E, Ross S, Delisle MF, Amankwah K, Guselle P, Gafni A, Lee SK,Armson BA; MACS Collaborative Group. Multiple courses of antenatal corticosteroids for preterm birth (MACS): a randomized controlled trial. Lancet 2008;372(9656):2143-51. 